EPJ Web of Conferences 37, 09022 (2012)

DOI: $10.1051 /$ epjconf/20123709022

(C) Owned by the authors, published by EDP Sciences, 2012

\title{
Kaon and strangeness production in an effective relativistic mean field model
}

\author{
A. Lavagno and D. Pigato
}

Department of Applied Science and Technology, Politecnico di Torino, I-10129 Torino, Italy and Istituto Nazionale di Fisica Nucleare (INFN), Sezione di Torino, I-10126 Torino, Italy

\begin{abstract}
We investigate the strangeness production at finite temperature and baryon density by means of an effective relativistic mean-field model with the inclusion of the full octet of baryons and kaon mesons. Kaons are considered taking into account of an effective chemical potential depending on the self-consistent interaction between baryons. In this context, we study the influence of the kaon-nucleon interaction in the determination of the kaon to anti-kaon ratio and in the strangeness production. The results are then compared with a minimal coupling scheme, calculated for different values of the antikaon optical potential.
\end{abstract}

We investigate the nuclear medium in the context of relativistic mean field approach, where the nuclear force is mediated by the exchange of isoscalar-scalar $(\sigma)$, isoscalar-vector $(\omega)$ and isovectorvector $(\rho)$ mesons fields [1,2]. The hadronic lagrangian can be expressed as: $\mathcal{L}_{H}=\mathcal{L}_{\text {octet }}+\mathcal{L}_{K}$, where $\mathcal{L}_{\text {octet }}$ stands for the full octet of baryons in the quantum hadrodynamics model with the GM3 parameters set [1] and $\mathcal{L}_{K}$ for kaon mesons (see below for details). Hyperon degrees of freedom are included taking into account of the determination of the corresponding meson-hyperon coupling constants that have been fitted to hypernuclear properties $[3,4]$.

Because we are going to describe finite temperature and density nuclear matter with respect to strong interaction, we have to require the conservation of three "charges": baryon number, electric charge and strangeness number. Therefore, the chemical potential of particle of index $i$ can be written as

$$
\mu_{i}=b_{i} \mu_{B}+c_{i} \mu_{C}+s_{i} \mu_{S},
$$

where $b_{i}, c_{i}$ and $s_{i}$ are, respectively, the baryon, the electric charge and the strangeness quantum numbers of the $i$-th hadrons and the effective chemical potential $\mu_{i}^{*}$ of the $i$-th baryon is given by $\mu_{i}^{*}=\mu_{i}-g_{\omega i} \omega-g_{\rho i} \tau_{3 i} \rho$.

In this work, we are going to study the kaon degrees of freedom using two different approaches. At this scope, for simplicity, other strangeless mesons (mainly pions) are not considered in our analysis, assuming that they do not sensibly affect the strangeness production but contribute essentially to the total pressure and energy density. Heavier strange meson degrees of freedom have been also neglected.

In the first approach, we consider the interaction between kaons and baryons by means of a direct minimal coupling scheme with the meson fields [4-7]. In this scheme the kaon lagrangian density can be written as

$$
\mathcal{L}_{K}=D_{\mu}^{*} \Phi^{*} D^{\mu} \Phi-m_{K}^{* 2} \Phi^{*} \Phi
$$

where $D_{\mu}=\partial_{\mu}+i g_{\omega K} \omega_{\mu}+i g_{\rho K} \tau_{3 K} \rho_{\mu}$ is the covariant derivative of the meson field, $m_{K}^{*}=m_{K}-g_{\sigma K} \sigma$ is the effective kaon mass and $\tau_{3 K}$ is the third component of the isospin operator.

The kaons vector and iso-vector coupling constant, are obtained from the quark model and the isospin counting rules and set equal to $g_{\omega K}=g_{\omega N} / 3$ and $g_{\rho K}=g_{\rho N}$. Whereas the scalar $g_{\sigma K}$ coupling constant is determined from the study of the real part of the anti-kaon optical potential by setting $U_{K^{-}}=-g_{\sigma K} \sigma-g_{\omega K} \omega$ at the saturation nuclear density and in a symmetric nuclear matter.

This is an Open Access article distributed under the terms of the Creative Commons Attribution License 2.0, which permits unrestricted use, distribution, and reproduction in any medium, provided the original work is properly cited. 

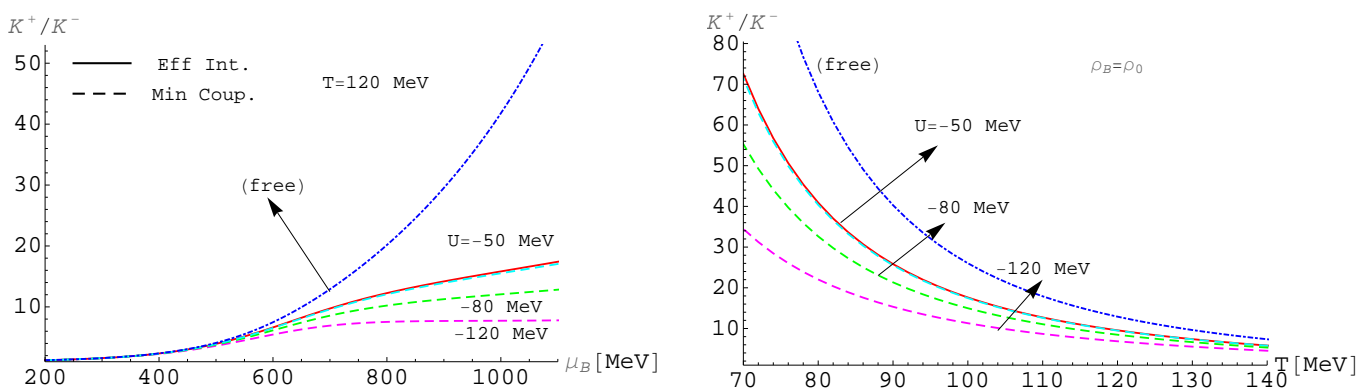

Fig. 1. Left panel: Kaon to anti-kaon ratio as a function of baryon chemical potential $\left(\mu_{B}\right)$ at a fixed temperature $(T=120 \mathrm{MeV})$ for different values of anti-kaon optical potential and in the effective relativistic mean-field model with effective kaon chemical potentials. Right panel: Kaon to anti-kaon ratio as a function of temperature at a fixed baryon density $\left(\rho_{B}=\rho_{0}\right)$. The dot-dashed lines correspond to the contribution with noninteracting kaons.

In this investigation we fix the anti-kaon optical potential equal to $U_{K^{-}}=-50 \mathrm{MeV},-80 \mathrm{MeV}$ and $-120 \mathrm{MeV}$, on the basis of recent theoretical calculations and experimental measurements $[4,6,8-$ 13]. For these values of the anti-kaon potential depth, we obtain the following kaon optical potentials $\left(U_{K^{+}}=-g_{\sigma K} \sigma+g_{\omega K} \omega\right.$, where the sign + in the $\omega$-field, is due to the G-parity): $U_{K^{+}}=47 \mathrm{MeV}, 17$ $\mathrm{MeV}$ and $-23 \mathrm{MeV}$, respectively for $U_{K^{-}}=-50 \mathrm{MeV},-80 \mathrm{MeV}$ and $-120 \mathrm{MeV}$, at the saturation nuclear density. In this study we neglect the contribution of the neutral kaons ( $K^{0}$ and $\bar{K}^{0}$ ) because of the large uncertainty in literature on their coupling constants with the meson fields.

The second approach, consist in an alternative formulation, based on the self-consistent interaction between baryons [3,7]. In this scheme mesons are treated as a quasi ideal Bose gas with an effective chemical potential $\mu^{*}$, obtained from the bare one given in Eq.(1) and subsequently expressed in terms of the corresponding effective baryon chemical potentials, respecting the strong interaction. More explicitly, the kaons and anti-kaon effective chemical potential can be written as

$$
\begin{aligned}
& \mu_{K^{+}}^{*}=\left(\mu_{p}^{*}-\mu_{\Lambda}^{*}\right)=\mu_{p}-\mu_{\Lambda}-\left(1-x_{\omega \Lambda}\right) g_{\omega N} \omega-\frac{1}{2} g_{\rho N} \rho, \\
& \mu_{K^{-}}^{*}=\left(\mu_{\Lambda}^{*}-\mu_{p}^{*}\right)=\mu_{\Lambda}-\mu_{p}+\left(1-x_{\omega \Lambda}\right) g_{\omega N} \omega+\frac{1}{2} g_{\rho N} \rho,
\end{aligned}
$$

where $x_{\omega \Lambda}=g_{\omega \Lambda} / g_{\omega N}$. Thus, the effective meson chemical potentials are coupled with the meson fields related to the interaction between baryons. This assumption can be seen somehow in analogy with the hadron resonance gas within the excluded-volume approximation. There the hadronic system is still regarded as an ideal gas but in the volume reduced by the volume occupied by constituents (usually assumed as a phenomenological model parameter), here we have a (quasifree) kaon gas but with an effective chemical potential that contains the self-consistent interaction of the meson fields.

Finally, at finite temperature the thermodynamical quantities can be obtained in the standard way from the total grand potential $\Omega_{H}=\Omega_{B}+\Omega_{K}$ for the two different approaches.

We are going to report the most relevant results obtained from a comparative study of kaons and strangeness production in the two aforementioned models and for different values of the anti-kaon optical potential. In the left panel of Fig. 1, we show the kaon to anti-kaon ratio as a function of the baryon chemical potential $\left(\mu_{B}\right)$ in the effective mean field model and in the chiral minimal coupling one, for different choices of $U_{K^{-}}$. It is interesting to observe that the results obtained in the minimal coupling scheme for a moderate potential depth (for values $U_{K^{-}} \simeq-50 \mathrm{MeV}$, as suggested by recent self-consistent calculations based on chiral Lagrangian [11,12] and coupled-channel G-matrix theory [10]) are very close to those of the effective relativistic mean field model. Furthermore, let us observe that, for strong attractive optical potential $\left(U_{K^{-}}=-120 \mathrm{MeV}\right)$ and for $\mu_{B}>750 \mathrm{MeV}\left(\rho_{B} \geq \rho_{0}\right)$, the $K^{+} / K^{-}$ratio becomes constant, whereas it continues to grow for smaller values of $U_{K^{-}}$and in the effective mean field model.

In the right panel of Fig. 1, we report the trend of the kaon to anti-kaon ratio as a function of the temperature, at fixed $\rho_{B}=\rho_{0}\left(\mu_{B} \approx 600-700 \mathrm{MeV}\right.$, depending on the particular choices of 

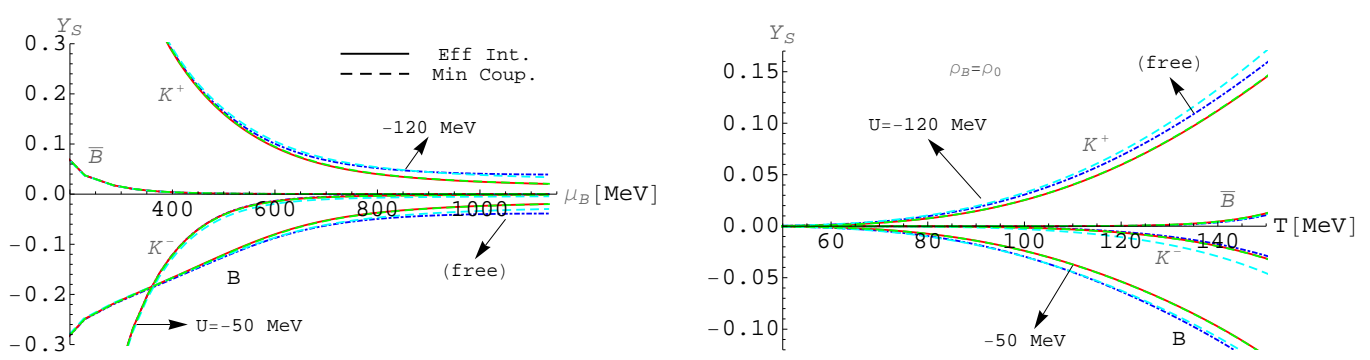

Fig. 2. Left panel: Strangeness concentration $Y_{S}$ as a function of baryon chemical potential $\left(\mu_{B}\right)$ at a fixed $T=120$ $\mathrm{MeV}$. The solid lines correspond to the results obtained in the effective relativistic mean field model, the dashed lines to different values of anti-kaon optical potential and the dot-dashed line to the free kaon case. In the right panel we report the strangeness concentration as a function of the temperature at fixed baryon density $\left(\rho_{B}=\rho_{0}\right)$.

$U_{K^{-}}$) in the effective mean field model and for different values of $U_{K^{-}}$. We observe again a good correspondence between the effective mean field model and the minimal coupling scheme for $U_{K^{-}}=$ $-50 \mathrm{MeV}$. Moreover, let us observe that, in absence of meson fields interaction, the ratio is sensibly increased, especially at high $\mu_{B}$ and at low temperature.

Finally, in Fig. 2, we show the strangeness concentration $Y_{S}$ of hyperons and kaons as a function of the baryon chemical potential at the fixed temperature $T=120 \mathrm{MeV}$ (left panel) and as a function of the temperature at the fixed baryon density $\rho_{B}=\rho_{0}$ (right panel). We report the case of $U_{K^{-}}=-50$ $\mathrm{MeV}$ and $U_{K^{-}}=-120 \mathrm{MeV}$ only. As we can see at moderate values of the anti-kaon optical potential the results obtained within the minimal coupling scheme become practically the same of the effective relativistic equation of state. This behavior persists by increasing of the temperature (right panel of Fig. 2), in particular, strange particles start to be abundantly produced above $T=100 \mathrm{MeV}$, whereas, at lower temperatures, due to the small fraction of strangeness production, they are negligible. In absence of interaction (dot dashed line) the kaon concentration is close to that of the relativistic mean field model at low $\mu_{B}$, whereas at high $\mu_{B}$, it converges to the minimal coupling scheme at $U_{K^{-}}=-120$ $\mathrm{MeV}$. These behaviors, could be considered as a relevant feature for the determination of the real part of the anti-kaon optical potential, for example in the future CBM (compressed baryonic matter) experiment of the FAIR (Facility of Antiproton and Ion Research) project [14].

\section{References}

1. $\quad$ N.K. Glendenning and S.A. Moszkowski, Phys. Rev. Lett. 67 (1991) 2414

2. L. Bonanno, A. Drago, A. Lavagno, Phys. Rev. Lett. 99 (2007) 242301

3. A. Lavagno, Phys. Rev. C 81 (2010) 044909

4. N.K. Glendenning and J. Schaffner-Bielich, Phys. Rev. C 60 (1999) 025803

5. R. Cavagnoli, C. Providencia, D.P. Menezes, Phys. Rev C 83 (2011) 045201

6. S. Banik, W. Greiner and D. Bandyopadhyay, Phys. Rev C 78 (2008) 065804

7. F. Iazzi et al., Int. J. Mod. Phys. E 21 (2012) 1250028

8. E. Friedman, A. Gal, J. Mares and A. Cieply, Phys. Rev. C 60 (1999) 024314

9. J.A. Pons, S. Reddy, P.J. Ellis, M. Prakash and J.M. Lattimer, Phys. Rev. C 62 (2000) 035803

10. L. Tolos, A. Ramos and A. Polls, Phys. Rev. C 65 (2002) 054907

11. M. Lutz and C.L. Korpa, Nucl. Phys. A 700 (2002) 309

12. M. Lutz and E.E. Kolomeitsev, Nucl. Phys. A 700 (2002) 193

13. A. Gal, Nucl. Phys. A 691 (2001) 268

14. P. Senger, J. Phys. G: Nucl. Part. Phys. 30 (2004) S1087; P. Senger et al., ibid. 36 (2009) 064037; W.F. Henning, Nucl. Phys. A 805 (2008) 502c 